\title{
Panorama histórico de la Ciencia y la Tecnología
}

Jorge Salguero Cubides *

\section{Resumen}

En el artículo se desarrolla una reflexión alrededor de unas definiciones de ciencia, de ciencias básicas y aplicadas, de la clasificación de las disciplinas científicas y el método científico como fundamento para la construcción de leyes y teorías. Se hace énfasis en el progreso de la ciencia, con sus autores, descubrimientos e invenciones a través de los siglos, desde que el hombre apareció sobre la Tierra y empezó a utilizar su inteligencia para crear ciencia y el uso de sus manos para construir máquinas e instrumentos que fue inventando como aplicación a los conocimientos adquiridos, originando la tecnología.

Palabras claves: Ciencia, tecnología, método científico, desarrollo histórico.

\section{Abstract}

This article makes a reflection on some definitions of science, such as basic and applied sciences, the classification of scientific disciplines, and the scientific method as a basis for building laws and theories.

It emphasises the progress of science and its authors, discoveries and inventions through the centuries since man appeared on the earth's surface and started using his mind to create science and his hands to build machines and invented tools as an application of the knowledge acquired, making technology possible.

La Historia de la ciencia estudia el desarrollo de los conocimientos científicos y tecnológicos de las sociedades humanas. Estudia también el impacto que la ciencia y la tecnologia han tenido históricamente en la cultura, la economia y la política.

La palabra "ciencia" etimológicamente proviene del latín "scientia", conocimiento el equivalente del griego "episteme", conocimiento riguroso, metódico, organizado. La ciencia es un conjunto de los conocimientos adquiridos por el hombre a lo largo de la historia, debidamente ordenados, según sus principios, causas, métodos y técnicas y clasificados según las distintas ramas del saber. La aplicación de esos métodos y conocimientos conduce a la generación de más conocimiento objetivo en forma de predicciones concretas, cuantitativas y comprobables referidas a hechos observables, pasados, presentes y futuros.

La ciencia puede diferenciarse en ciencias básicas y aplicadas. Las ciencias básicas, se orientan a la aplicación de nuevos conocimientos, responden a demandas internas de la propia disciplina, estudian problemas definidos. Las ciencias aplicadas, estudian la aplicación del conocimiento científico a las necesidades humanas y al desarrollo tecnológico.

\section{Disciplinas científicas}

El epistemólogo alemán Rudolf Carnap en su libro "Fundamentos de lógica matemática" fue el primero en clasificar la ciencia en:

Ciencias formales: Son aquellas ciencias que no estudian fenómenos empíricos. Utilizan la deducción como método de búsqueda de la verdad: Lógica-Matemática.

Ciencias naturales: En ellas se ubican las ciencias que tienen por objeto el estudio de la naturaleza. Siguen el mé- 
todo científico: Astronomía, Biología, Fisica, Química, Geología y Geografia Fisica.

Ciencias sociales: Son todas las disciplinas que se ocupan de los aspectos del ser humano- cultura y sociedad. El método depende de cada disciplina en particular. Antropología, Demografia, Economia, Historia, Psicología, Sociología y Geografía humana.

\section{Método} científico

El método científico ( del griego:

meta $=$ hacia, a lo largo odos= camino hacia el conocimiento) se concibe como una estructura, un armazón formado por reglas y principios claramente ordenados.

El método cientifico es el proceso mediante el cual una teoria cientifica es validada o descartada. Existe una serie de pasos inherentes al proceso científico, que son respetados en la construcción y desarrollo de nuevas teorías. Estos son:

1. Observación: Consiste en la recolección de hechos acerca de un problema o fenómeno natural o social que despierta nuestra curiosidad. Las observaciones deben ser lo más claras y numerosas posibles, porque han de servir como base de partida para la solución.

2. Descripción: Se trata de una detallada descripción del fenómeno objeto de estudio.
3. Hipótesis: Es la pregunta o preguntas que hacemos ante el hecho observado o descrito. La hipótesis en el sentido original de la palabra, se entiende como suposición o conjetura acerca de algún fenómeno u objeto de estudio principal a delimitar, el problema a investigar, teniendo en cuenta algunas variables que se refieren a las caracteristicas propias del fenómeno investigado.

4. Experimentación: Consiste en la verificación o comprobación de la hipótesis. La experimentación determina la validez de las posibles explicaciones que nos hemos dado y decide que una hipótesis se acepte o se rechace, La experimentación no es repetible a todas las ramas de la ciencia, especialmente en ciencias humanas, pero lo es, necesariamente, en los fenómenos naturales.

5. Registro y análisis de datos: La labor cientifica necesita la recolección de datos (observaciones iniciales, durante el final del experimento) en forma organizada, de manera que sea posible determinar relaciones entre estos, para lo cual se utilizan tablas, cuadros, gráficas, etc.

6. Análisis de resultados:Los investigadores someten la información a muchos estudios, entre estos al análisis estadistico, que consiste en utilizar las matemáticas como función en la expresión de modelos científicos. 


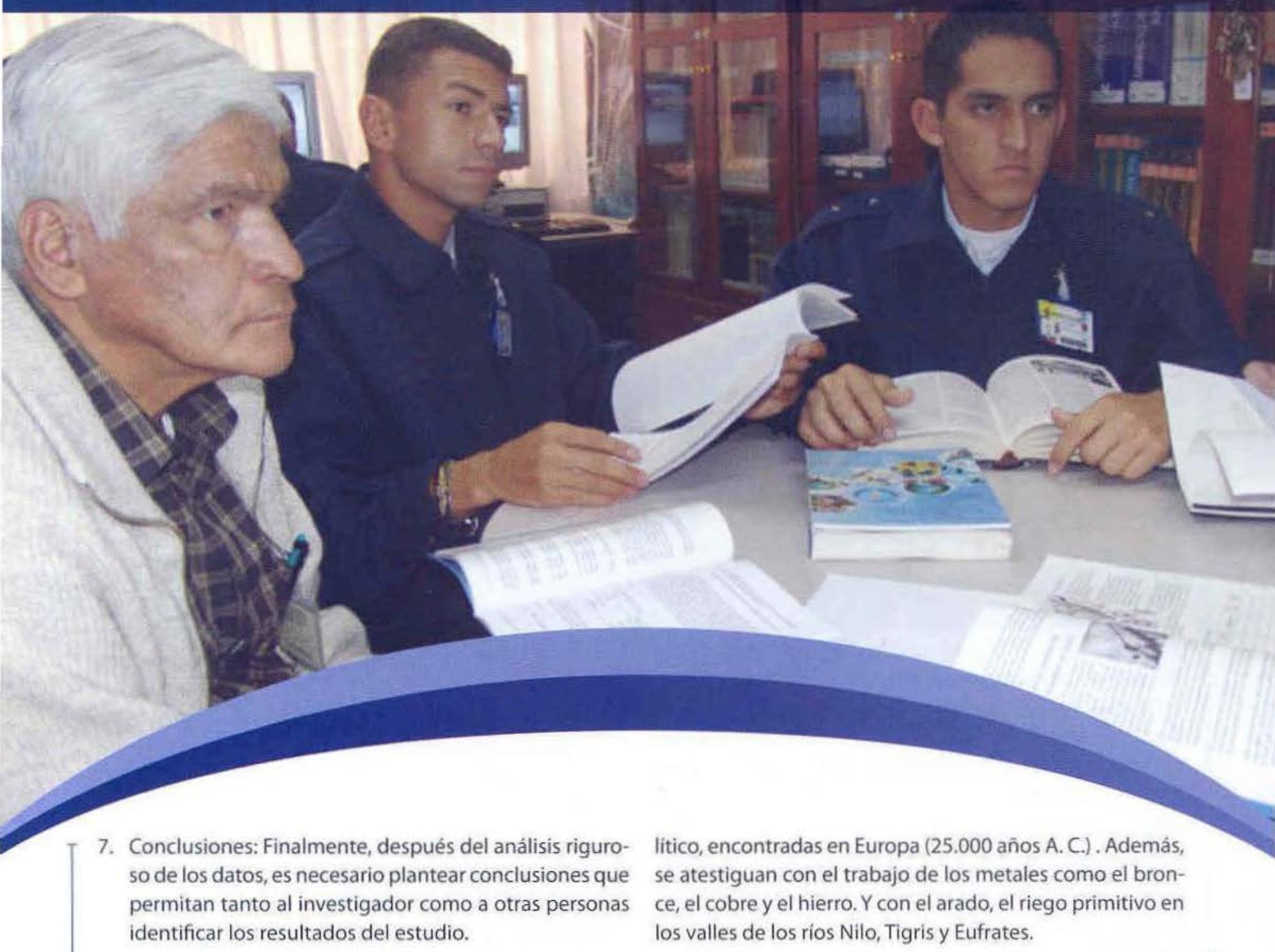

Los grandes proyectos investigativos se realizan en centros de investigación y universidades en forma multidisciplinaria, con importantes inversiones en instalaciones, e involucrando a una gran cantidad de científicos de diferentes paises y especialidades. Para que todos puedan colaborar unidos con eficiencia necesitan un método sistemático, que se pueda repetir o comprobar en cualquier parte del mundo.

\section{Desarrollo histórico breve de la ciencia y la tecnología}

Desde los tiempos prehistóricos los conocimientos fueron trasmitidos de generación en generación por medio de la tradición oral. La sistematización del conocimiento desde aquellos tiempos se comprueba en los dibujos que los pueblos pintaban en las paredes de las cuevas, el uso del fuego y las hachas manuales de piedra, del periodo paleo-

Después del año 4.000 A.C., apareció una de las creaciones más complejas de la humanidad: la ciudad, con la construcción de palacios, templos, tumbas y amurallamientos. Se construyen las pirámides de Egipto, México, y también centros rituales como Machu Pichu en el Perú, que simbolizan el poder organizativo y la magnitud tecnológica de los centros urbanos.

Los griegos y los romanos fueron grandes tecnólogos en cuanto a la organización y la construcción de palacios, templos y edificaciones. El crecimiento de las ciudades también estimuló la invención de la escritura, inicialmente en Mesopotamia con la escritura cuneiforme sobre tablillas que datan de los 2000 A.C., y en Egipto con los jeroglíicos.

De los hombres de esa época se destacan los filósofos jonios (siglo VI A.C.) que sustituyen las representaciones antropomórficas de los mitos por elementos de la naturaleza y elaboran cosmogonías de perfil científico - filosófi- 
co. Sus representantes más sobresalientes son el filósofo Tales de Mileto que introdujo el concepto de que la tierra era un disco plano, Anexágoras matemático y Demócrito que tendrá notable influencia sobre la medicina de Hipócrates. Además sobresalen el matemático y filósofo Pitágoras con su teorema, en astronomía Tholomeo, autor de la teoría geocéntrica quien sostenía que la tierra era el centro del universo.

En Atenas, en el siglo IV A.C., Aristóteles en su pensamiento destaca la teoria de las ideas, que proponía que los objetos del mundo físico eran perfectos, y que solo las formas perfectas pueden ser objeto del verdadero conocimiento.

En la edad media, periodo histórico transcurrido entre la caída de Roma y el renacimiento (aproximadamente del año 400 al 1500 D. C.), en contra de las creencias populares, se produjeron grandes avances tecnológicos. Las culturas bizantina e islámica que prosperaron en esta época tuvieron una importante actividad en las áreas de la filosofia, el arte, la literatura, la religión; en especial la cultura islámica que aportó numerosas contribuciones científicas, que tendrán gran importancia en el renacimiento europeo, por ejemplo en el arte de la guerra, la agricultura, el transporte, la imprenta, el astrolabio, la pólvora, la cartografía que contribuyeron a los grandes descubrimientos geográficos del siglo XV y XVI.

El renacimiento (S.XV), llamado asi por el renacer de las ciencias y las artes griegas y latinas, marcó el fin de la edad media y creó elementos sólidos en el desarrollo de nuevos conocimientos. De los científicos de esta época se destaca Nicolás Copérnico quien inició la revolución científica con su teoría heliocéntrica. Entre los pensadores más prominentes se destacan Francis Bacon en Inglaterra, René Descartes en Francia y Galileo Galilei en Italia.

El renacimiento y la ilustración cambian la postura de los hombres hacia la tecnologia, considerando que ésta es intrinsicamente buena y que una mala utilización de la misma puede causar efectos indeseables. Bacon, en el siglo $X V I I$, fue uno de los primeros científicos en rechazar la idea de que la técnica ejercia una influencia corruptora sobre la moral al contrario, defendió que los hombres encontrarian en la tecnología la forma de mitigar el sufrimiento propio de la condición humana.

Del siglo XVII y XVIII sobresalen científicos como Isaac Newton quien aportó la teoria de la ley de gravitación universal en 1687; Gottfried Leibniz que sentó las bases de la ciencia y las matemáticas actuales; y René Descartes, filósofo francés, quien dio paso a la ciencia materia- lista del siglo XVIII. El químico francés Antoine Laurent de Lavoisier publicó el "Tratado elemental de Química" en 1789 e inició la revolución de la química cuantitativa. Y Charles Darwin, quien desarrolló la teoría de la evolución de las especies.

La sociedad moderna se inicia con la revolución industrial producida a lo largo de los siglos XVIII y XIX. La industria se convierte en el motor de la actividad económica y las herramientas artesanales son sustituidas por las máquinas (máquina de vapor, máquinas eléctricas y nuevas tecnologías) que modifican los sistemas de producción, transporte y comunicación.

En los siglos XIX y XX se han producido grandes avances científicos como el mapa del genoma humano, el desarrollo de la bomba atómica, los viajes espaciales, el avance de la medicina, vacunas contra la malaria, la fiebre amarilla, la ingeniería, las comunicaciones, etc. Estamos en una constante evolución gracias a investigadores que estudian y realizan estos grandes descubrimientos.

Las revoluciones cientificas de principios del siglo XX estuvieron ligadas especialmente al campo de la fisica a través del desarrollo de la mecánica quántica y la teoría de la relatividad de Albert Einstein y en el siglo XXI la ciencia se enfrenta a las revoluciones de la biotecnología y nanotecnología.

La tecnología se ha situado entre la ciencia y la técnica, entre los componentes teóricos de la ciencia y las ejecuciones prácticas de la técnica, siempre orientado a la resolución de los problemas que la humanidad se ha ido encontrando, aunque en algunos momentos se replanteen las dimensiones éticas de los avances conseguidos.

\section{BIBLIOGRAFIAA}

Bunge Mario. La ciencia su método y su filosofia. Editorial Alianza, 1995.

Castells Manuel. Trilogía. La era de la información. Editorial Alianza . Barcelona 1986

Kuhn, Thomas. La estructura de las revoluciones cientificas. 1962

www. monografías. com. La ciencia y la tecnología en el contexto del siglo XXI.2007

www. La ciencia. monografias.com 2006

www. Ciencia. Wikipedia, la enciclopedia libre. 2007 